\title{
FRAMING A WELL-STRUCTURED SINGLE BEST RESPONSE MULTIPLE CHOICE QUESTIONS (MCQS) - AN ART TO BE LEARNED BY A TEACHER
}

\author{
Chaudhary $\mathbf{N}^{1}$, Bhatia BD ${ }^{2}$, Mahato SK ${ }^{1}$
}

\section{INTRODUCTION}

MCQs are most widely used objective tests also called an Item. In a single best response MCQ, a student has to chooseone answer from a number of choices supplied.MCQs are objective questions to assess some aspects of student's learning toassessknowledge, comprehension and application. It can also be used to assess higher order skills such as evaluation and synthesis.

It is a false belief that a multiple choice question is suitable for testing factual information only,whereas essay type questions can test higher-order cognitive skills. A poorly-written multiple choice items elicit complaints from students that the questions were not clear or choices were not appropriate. If the teacher follows special requirements for writing multiple choice questions and also tests its reliability and validity, awell-designed multiple choice item can test even high levels of student learning skills including all six levels of Bloom`s Taxonomy of cognitive objectives (See table).

\section{Table1 : Levels of cognitive learning (Blooms taxonomy of cognitive objectives, 1956)}

\section{Levels of cognitive learning}

1. Knowledge: Simple recognition or recall of material

2. Comprehension: Restating or reorganizing material to show understanding

3. Application: Problem solving or applying ideas in new situations

4. Analysis: Separating ideas into component parts, examining relationships

5. Synthesis: Combining ideas into statement or product new to the learning

6. Evaluation: Judging by using self-produced criteria or established standards

1. Assistant Professor, Department of Pediatrics, Universal College of Medical Sciences \& Teaching Hospital Bhairahawa, Nepal

2. Medical Superintendent, Professor \& Head, Department of Pediatrics, Universal College of Medical Sciences \& Teaching Hospital Bhairahawa, Nepal

\author{
For Correspondence \\ Dr.Nagendra Chaudhary M.B.B.S., M.D. \\ Assistant Professor, \\ Department of Pediatrics \\ Universal College of Medical Sciences \& Teaching Hospital \\ Bhairahawa, Nepal \\ Email: enagendra@hotmail.com
}




\section{THE NEED FOR MCQs}

Marking large numbers of student on essay type questions, projects and other subjective assessments is time-consuming, labour intensive and prone to errors of consistency which is quite wide. Including MCQ`s during assessment allows for more objective assessment of students and is time saving (OMR assessment or staff members with no special knowledge in the area can also check).

\section{Introduction to single best response multiple choice item}

A multiple-choice question or item comprises of following

- Directions to students

- Stem - the text of the question; which presents the problem

- Key: the correct answer in the list of options provided

- Distracters: the incorrect answers in the list of options

\section{The stem of an item can present the problem in one or more} formats

a) Complete question: e.g., Which of the following is the most sensitive test for enteric fever in the first week of disease?
(1) Stool culture
(2) Blood culture
(3) Urine culture
(4) Widal test

b) Incomplete statement: e.g., The most sensitive test for enteric fever in the first week of disease is:
(1) Stool culture
(2) Blood culture
(3) Urine culture
(4) Widal test

Students are supposed to select the correct response (key) from the list of alternatives/distractors. Construction of meaningful and worthwhile multiple choice item is difficult and time consuming. Thorndike and Hagen mentioned that "An ingenious and talented item writer can construct multiple choice items that require not only the recall of knowledge but also the use of skills of comprehension, interpretation, application, analysis, or synthesis to arrive the "keyed" answer.

Wilbert J. Mckeachiehas said that "the greater you experience in their construction, the longer it takes per item to construct a reasonably fair, accurate and inclusive question". In other words, as you get better in construction, things may appear more difficult.

\section{ADVANTAGES AND DISADVANTAGES OF MCQs}

\section{Advantages}

1. Precise and unambiguous measurement of intellectual process

2. Reduces marking time, easy to evaluate

3. Increases range and variety of facts to be tested in a given time.

4. Analysis of individual questions is possible

5. Provision of automatic feedback to student (when used in computer-based assessment) or detailed feedback after the test (Excellent basis for post-test discussion, why the distractors are wrong and why the key is correct)

6. Potential for more frequent assessment

7. Questions can be pre-tested in order to evaluate their effectiveness and level of difficulty

8. Can assess the ability to integrate information from different sources

9. MCQs are useful in diagnosing students difficulties, if the distractors are written to reveal common errors

10. Ensures objectivity, specificity, reliability, validity.

11. Preparation involves all the colleagues and their positive criticism during discussion

12. For students- the advantage is easy to score, if they have read even once, the recall memory may help.

\section{Disadvantages}

- Significant amount of time required to construct a good quality multiple choice question

- Writing questions that test higher order skills requires much more effort and brain storming sessions

- Cannot assess the ability to write logically and systematically and capability of expression

- Cannot be used to assess integration of ideas and the capacity to develop a sustained argument

- Not suited to test highest level of cognitive domain like synthesis and evaluation

- Cannot test the motor skills like communication, and psychomotor skills 
- Provides clues and scope for guess work (although this can be minimized by negative marking)

\section{SUGGESTIONS FOR CONSTRUCTING A GOOD QUALITY MULTIPLE-CHOICE QUESTIONS}

\section{DIRECTIONS}

Instruction to candidate which could be common for set of similar questions. eg., In case of single best response type, the direction could be "Select the most appropriate answer and darken the corresponding circle in answer sheet provided".

\section{STEM}

The basic rule for stem-writing is that students should be able to understand the question without reading it several times and without having to read all the options. Following precautions should be observed while writing stem:

a) Present stem as a single, definite statement to be completed or answered by one of the several given choices as alternatives. One of the alternatives is the key and others are functional distractors.

b) Avoid unnecessary and irrelevant statements.

c) Use clear, straightforward language in the stem of the item. Questions that are constructed using complex wording may become a test of reading comprehension rather than an assessment of whether the student knows the subject matter or not.

d) Use negatives sparingly. If negatives are to be used, capitalize, underscore embolden or otherwise highlight. Do not use double negatives e.g., "Which of these is not the least important clinical presentation in Dengue?"

\section{Double negatives will only confuse the student.}

e) Put as much of the question in the stem as possible, rather than duplicating material in each of the options. All common elements are to be placed in the stem.

f) Never, always, usually, sometimes etc. should be used with great caution.

g) Vague expressions like fairly high, considerably greater etc. should be avoided.

h) Stem should not ask for an opinion. i) NOT or EXCEPT should be capital underlined.

\section{DISTRACTORS}

a) For single response MCQs, ensure that there is only one correct response.

b) Use only plausible and functional alternatives as distracters.

c) Avoid giving clues to the correct answer eg., All distractors should be of similar length, numerical observations in similar range etc.

d) If possible, avoid the choices "All of the above" and "None of the above". If you do include them, make sure that they appear as correct answers at some of the time.

e) Distracters based on common student errors or misconceptions are very effective.

f) Do not create distracters that are so close to the correct answer that they may confuse student who even really knows the answer to the question.

g) Provide a sufficient number of distracters: Use three, four or five alternatives in a multiple-choice questions. It is thought that three distracters are necessary for the item to be suitably difficult (Owen and Freeman, 1987).Higher the number of distracters, the less likely it is for the correct answer to be chosen through guess work.

h) Avoid abbreviations and acronyms.

i) For items with numerical alternatives, arrange them in a rank order. eg,. 5, 10, 15, 20 (not 15, 5, 20, 10). If range is given, it should be same in all dimensions $(1-5,6-10,11-$ $15,16-20)$.

j) All distractors should be discussed by group of teachers or examiners for unambiguity and also should be tried on a group of students before giving in examination.

k) All distractors should be functional and should be by its nature, content, be such that it appears to have something to do with stem. Errors from students' answers and their misconceptions are the best distractors.

\section{KEY}

a. It should undeniably be the correct response.

b. Is the key to an item provided by data in another item? 
c. Care should be taken not to use words in key which are synonyms or very similar to words in stem.

Following are some of the single best response type of MCQs set by teachers in a group discussion. Identify the defect in these MCQs. (The defects shall be discussed in the next issue of Journal).

1. Which of the following are not loop diuretics EXCEPT:
a) Furosemide
b) Chorthalidone
c) Aldactone
d) Dytide

2. An intercostal tube may be placed in a patient with flail chest but without pneumothorax or hemothorax, to:
a) remove air
b) Remove blood
c) Prevent development of tension pneumothorax

3. Protein requirement of Indian reference man is:
a) 1 g per kg of body weight
b) 2 g per kg of body weight
c) 3 g per kg of body weight
d) 4 g per kg of body weight

4. Wilms` tumour:

a) Congenital tumour

b) Congenital renal tumour

c) Congenital supra renal tumour

d) Congenital tumour of sympathetic chain

5. With advancement of statistical methods there has been concurrent simplification of diagrammatic representations. A suitable diagram for depicting data like monthly hospital admission rate would be:
a) Bar diagram
b) Histogram
c) Pie diagram
d) Scatter diagram
6. Following contrast media are used for IVP except
a) Conray
b) Urograffin
c) Dianosil
d) None of the above

7. Imipramine is an:
a) Sedative
b) Stimulant
c) Bronchodilator
d) Anti-depressant

8. Water hammer pulse is characterized by:
a) High volume
b) Rapid upstroke and descent of pulse wave
c) Rapid rate
d) Low pulse pressure

9. Protein content of buffalo milk ( $\mathrm{gm} / \mathrm{dl})$ is:
a) 1
b) 3
c) 2
d) 4.3

10. $\mathrm{ADH}$ is secreted by
a) Anterior pituitary
b) Posterior pituitary
c) Pars intermedia
d) Pars nervosa

\section{CHECK LIST IS GIVEN BELOW}

\section{Item as a whole}

a) Is the direction clear?

b) Is each item wholly independent of others?

c) Are all unrelated details eliminated?

d) Does the item test an important learning outcome/ important content area?

e) Is it specific?

f) Is it brief and not too enlarged?

g) Is the level of difficulty/discrimination appropriate?

h) What is the appropriate time for the item? 
i) Are there clues which suggest the correct answer?

j) Are negative statements used with care and appropriately emphasised?

k) Is the type of item the best one for the particular point or problem?

\section{Stem}

a) Is it clear, concise and unambiguous?

b) Does it ask for an opinion?

c) Is it a complete sentence or an idea but not merely a single word?

d) Have vague expressions like fairly high, considerably greater etc. been avoided?

e) Are all common elements placed in the stem?

f) Are words like never, always, usually, sometime etc. used with caution?

g) Are double negatives avoided?

\section{Options}

a) Are they logical and plausible?

b) Are they parallel and homogeneous?

c) Are options wherever appropriate arranged in numerical order?

d) Does all options complete the stem grammatically?

e) Are all distractors functional?

f) Is it ensured that when several different items are based on the same data it is not necessary for a candidate to answer one question correctly to obtain the correct answer to another?

g) Is care exercised in using all of the above/none of the above as distractors?

Key

a) Is it undeniably the only correct response? b) Are letters corresponding to the key equally distributed amongst options $\mathrm{a}, \mathrm{b}, \mathrm{c}, \mathrm{d}$ ?

c) Is key to an item provided by data in another item?

d) Is care taken not to use words in key which are synonyms or very similar to words in the stem?

e) Is the key too long to give a clue?

1. Bloom BS, Engelhart MD, Furst EJ, Hill WH, Krathwohl DR. Taxonomy of educational objectives: The classification of educational goals. Handbook I: Cognitive domain. New York: David McKay Company;1956.

2. Welsh AL. Multiple choice objective tests. In: Saunders P, Welsh $A$, Hansen WL, editors. Resource manual for teacher training programs in Economics. NY: Joint Council on Economic Education;1978. P. 191-228.

3. Cashin WE. Improving multiple-choice tests Idea Paper No. 16. Manhattan KS: Center for Faculty Development and Evaluation, Kansas State University; 1987. PMid: 3607376

4. Thorndike RL, Hagen E. Measurement and Evaluation in Psychology and Education. New York: John Wiley and Sons; 1969. PMid: 5794082

5. Gronlund NE. Measurement and Evaluation in Teaching 5th ed.New York:Macmillan Publishing Co.,Inc. 1985.

6. Educational testing service. Multiple choice questions: A closer look. Princeton NJ: Author 1973. 